\title{
Forecast analysis on satellites that need de-orbit technologies: future scenarios for passive de-orbit devices
}

\author{
Chiara Palla ${ }^{1}$ (D) Jennifer Kingston ${ }^{1}$
}

Received: 22 December 2015 / Accepted: 28 April 2016 / Published online: 10 May 2016

(C) The Author(s) 2016. This article is published with open access at Springerlink.com

\begin{abstract}
Propulsion-based de-orbit is a space-proven technology; however, this strategy can strongly limit operational lifetime, as fuel mass is dedicated to the de-orbiting. In addition previous reliability studies have identified the propulsion subsystem as one of the major contributors driving satellite failures. This issue brings the need to develop affordable de-orbit technologies with a limited reliance on the system level performance of the host satellite, ideally largely passive methods. Passive disposal strategies which take advantage of aerodynamic drag as the de-orbit force are particularly attractive because they are independent of spacecraft propulsion capabilities. This paper investigates the future market for passive de-orbit devices in LEO to aid in defining top-level requirements for the design of such devices. This is performed by considering the compliances of projected future satellites with the Inter Agency Space Debris Coordination Committee de-orbit time, to quantify the number of spacecraft that are compliant or non-compliant with the guidelines and, in this way, determine their need for the previously discussed devices. The study is performed by using the SpaceTrak ${ }^{\mathrm{TM}}$ database which provides future launch schedules, and spacecraft information; the de-orbit analysis is carried out by means of simulations with STELA. A case study of a passive strategy is given by the de-orbit mechanism technological demonstrator, which is currently under development at Cranfield University and
\end{abstract}

This paper is based on a presentation at the 5 th CEAS Air \& Space Conference, Sept. 7-11, 2015, Delft, The Netherlands.

Chiara Palla

c.palla@cranfield.ac.uk

1 Space Research Centre, Cranfield University, College Road, Cranfield, Bedfordshire MK43 0AL, UK designed to deploy a drag sail at the end of the ESEO satellite mission.

Keywords Space debris mitigation - Passive de-orbit device $\cdot$ Drag $\cdot$ Orbital decay $\cdot$ Future scenario

$\begin{array}{ll}\text { Abbreviations } \\ \text { AOCS } & \text { Attitude and orbit control system } \\ \text { ATP } & \text { Area time product } \\ \text { DOM } & \text { De-orbit mechanism } \\ \text { EoM } & \text { End of mission } \\ \text { ESEO } & \text { European Student Earth Orbiter } \\ \text { EBB } & \text { Elegant bread board } \\ \text { IADC } & \text { Inter-Agency Space Debris Coordination Committee } \\ \text { LEO } & \text { Low earth orbit } \\ \text { MOD } & \text { Mean of date } \\ \text { Req } & \text { Requirement } \\ \text { SDM } & \text { Space debris mitigation } \\ \text { SSO } & \text { Sun synchronous orbit } \\ \text { S/C } & \text { Spacecraft } \\ \text { TLE } & \text { Two line element } \\ Z & \text { Altitude }\end{array}$

\section{Introduction}

\subsection{Clean space}

Space debris represents a major risk to future space missions; this is why ESA, within the clean space initiative, is establishing requirements to mitigate the production of new space debris.

ESA started the clean space initiative in 2012 to redesign the way industry operates and ensures it operates in a safe and sustainable way both on terrestrial and space 
environments [1]. Clean space is implemented in four main branches: eco-design, green technologies, space debris mitigation, and technologies for space debris remediation.

Recent updates in the regulations on the safeguard of the Earth's orbital environment both by ESA [2] and the United Nations [3] show a progress in this area from guidelines to requirements.

\subsection{Space debris mitigation requirements}

The recent adoption of ISO 24113 Space System-space debris mitigation requirements [4] in the ESA Policy ESA/ ADMIN/IPOL(2014)2 ensures that a standard approach [5] on space debris mitigation requirements for ESA projects is established.

The International Standard defines two protected regions with regards to space debris as identified in the IADC mitigation guidelines [6]: low earth orbit (LEO) and geosynchronous earth orbit (GEO) regions.

LEO region is a spherical region that extends from the Earth's surface up to an altitude $(Z)$ of $2000 \mathrm{~km}$. GEO region $\left(Z_{\mathrm{GEO}}=35,786 \mathrm{~km}\right)$ is a segment of a spherical shell defined with a lower altitude equal to $Z_{\mathrm{GEO}}-200 \mathrm{~km}$, an upper altitude equal to $Z_{\mathrm{GEO}}+200 \mathrm{~km}$ and a latitude range $\left[-15^{\circ},+15^{\circ}\right]$.

The removal of spacecraft or orbital stages after the End of Mission from these two protected regions is required. In particular the probability of successful disposal of the space system shall be at least 0.9 when the disposal is executed (Req. 6.3.1.1).

Focussing on the LEO region, the main requirements about the space systems removal define the clearance time for de-orbit (Req. 6.3.3.1) and the disposal manoeuvre approach to be adopted after the EoM (Req. 6.3.3.2).

The time limit, in which the removal shall occur, is 25 years after the EoM; this is a result of the studies performed on space debris population in LEO [5] and a compromise between the debris growth and the cost burden for implementing mitigation measures.

After the EoM, the disposal manoeuvre shall be accomplished by one of the following approaches [4]:

(a) controlled re-entry,

(b) controlled manner into a targeted re-entry with a welldefined impact footprint on the surface of the Earth,

(c) controlled manner to an orbit with a shorter orbital lifetime (compliant with 6.3.3.1),

(d) by deploying a device augmenting the orbital decay so that the remaining orbital lifetime is compliant with the 25 years,

(e) natural orbital decay so that the remaining orbital lifetime is compliant with 6.3.3.1,

(f) controlled manner to an orbit with a perigee altitude sufficiently above the LEO protected region that long- term perturbation forces do not cause it to re-enter the LEO protected region within 100 years.

\subsection{De-orbit strategies}

The approaches identified in the LEO disposal requirement lead to different de-orbit strategies. The techniques to achieve the de-orbit can be grouped in two main categories: active and passive.

Active de-orbit strategies basically need continuous power supply together with AOCS to achieve the de-orbit. Among the active methods, there are on-board propulsion, electric propulsion, solid propulsion (e.g., smart propulsive device [7] currently at qualification level). On-board propulsion is a space-proven technology; however, this strategy can strongly limit operational lifetime, as fuel mass is dedicated to the de-orbiting (up to $20 \%$ of the spacecraft mass [8]). In addition, previous reliability studies have identified the propulsion subsystem as one of the major contributors driving satellite failures (see Fig. 1) [9]. It has also been seen that this subsystem experiences significant degradation on orbit and even when it (partially) fails, it is likely to have major effect on mission capability [10].

Furthermore, micro (10-100 kg) and smaller spacecraft are, in general, not provided with propulsion capabilities to achieve a controlled re-entry, so they need different deorbit disposal methods. Passive disposal strategies which take advantage of aerodynamic drag as the de-orbit force are particularly attractive because they are independent of spacecraft propulsion capabilities.

The drag force acts in the opposite direction of velocity, its effect is a change in semi-major axis and eccentricity of the orbit over time. It is only of second order magnitude as compared to the first order Earth's oblateness perturbation.

$F_{\text {drag }}=-\frac{1}{2} C_{\mathrm{d}} A \rho v^{2}$

where $C_{\mathrm{d}}$ is the coefficient of drag, $A$ is the surface area normal to the velocity vector, $\rho$ is the density of the atmosphere. For $700 \mathrm{~km}$ orbit, the order of magnitude is $10^{-6} \mathrm{~N} /$ $\mathrm{m}^{2}$, considering average atmospheric density value.

Drag devices increase the area-to-mass ratio of the spacecraft, augmenting the surface area normal to the velocity vector, and in this way they allow to reduce the natural orbit decay.

Three different concepts can be identified as drag augmentation devices, Nock describes them in [11] when considering the ATP:

1. Boom-supported film aerobrake also known as drag sail. It is a very thin film - the sail—stretched between support boom arms. For this option, Cranfield has own experience (e.g., Icarus1 [12] for the UK's TechDem- 
Fig. 1 Relative contribution of subsystems (gyro/reaction wheel, thruster/fuel, control processor, mechanisms/structures) to satellite failure, obtained from Weibull parameters of Castet study [9]

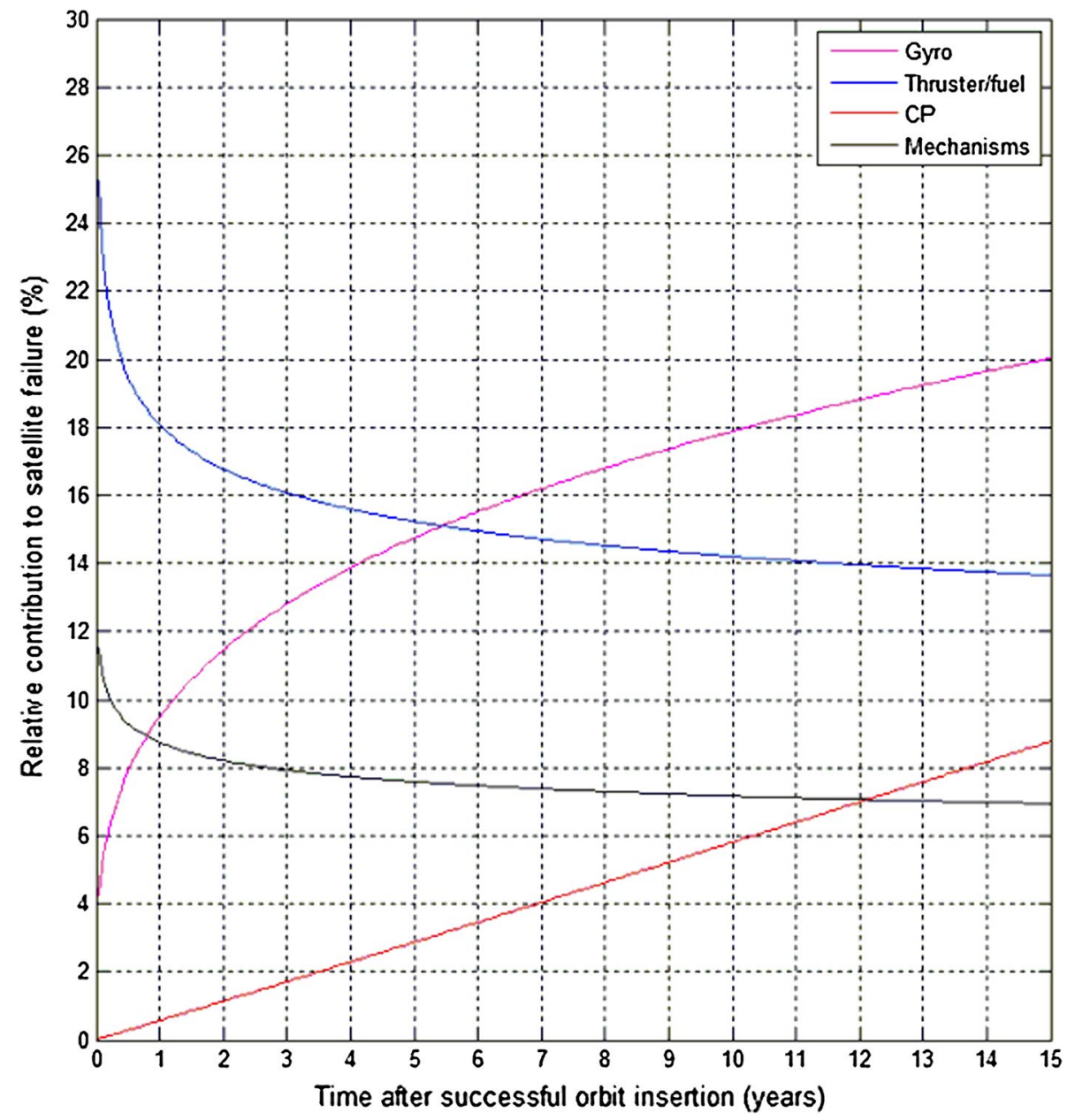

oSat-1 recently put on orbit) and on-going work for ESEO DOM; in addition there are other projects at late stage, such as AEOLDOS [13].

2. Inflation-maintained ultrathin envelope also identified as balloon. It requires gas, sensors, actuators and a controller as system elements for pressure variation during de-orbit.

3. Rigidizable space inflatable envelope. It is an envelope inflated and then rigidized through a mechanical or chemical process, similar to balloon concept.

Drag sails are promising, there is a consistent heritage in their design and current work in progress; in addition, they will lead to a reduction in debris collision risk as shown in [14].

\section{Methodology}

In this section, the tools, models, and workflow adopted to quantify the compliances of projected future satellites with the 25 years de-orbit time are presented. The study is performed by using the SpaceTrak ${ }^{\mathrm{TM}}$ database [15] which provides future launch schedules, and reliability and trend information for launch vehicles and spacecraft types; the de-orbit analysis is carried out by means of simulations with STELA, the semi-analytic orbit propagator designed by CNES [16].

The main phases of this work encompasses the evaluation of orbital data of satellites re-entered (TLE) with respect to STELA simulation; developing and updating of the future launch schedules database; division in satellite classes, types and orbits; performing STELA simulations for the de-orbit analysis of future satellites.

\subsection{Future launch database}

Spacetrak $^{\mathrm{TM}}$ provides an up-to-date database on satellites and launch vehicles and it has become the most used reference source for launch providers, satellite insurances, operators and manufacturers in the space sector.

For the purpose of this study, all the future satellites in LEO with a stated launch date (the range is 2015-2020) have been extracted; it is to be noted that suborbital 
Table 1 Summary of data available for the analysis on future launches 2015-2020 (last update April 2015)

\begin{tabular}{|c|c|c|c|c|}
\hline Group & $\begin{array}{l}\text { Total with } \\
\text { launch year } \\
\text { stated }\end{array}$ & $\begin{array}{l}\text { Data missing } \\
\text { for mass }\end{array}$ & $\begin{array}{l}\text { Data missing } \\
\text { for orbit }\end{array}$ & $\begin{array}{l}\text { Data miss- } \\
\text { ing for area }\end{array}$ \\
\hline $\begin{array}{l}\text { LEO (not } \\
\text { SSO/ } \\
\text { POLAR) }\end{array}$ & 143 & 19 & 31 & 39 \\
\hline SSO & 135 & 24 & 42 & 61 \\
\hline POLAR & 93 & 2 & 9 & 9 \\
\hline
\end{tabular}

Satellites are divided depending on the orbit type (group)

vehicles and cargo missions have not been considered since they are out of the objectives of this work.

Following the database classification, the satellites in LEO are divided into three different subgroups: polar orbit, sun-synchronous orbit (SSO), and LEO in general not fitting with the first two groups.

For each spacecraft in the sample, the key parameters collected from the database are: (1) S/C name, (2) S/C bus, (3) future launch date, (4) mass, (5) apogee, (6) perigee, (7) inclination.

Due to the uncertainty on the future launches and the information publicly available, the database is not "complete" for the orbit parameters and the spacecraft characteristics. For this reason, the missing data have been collected from different sources, the main ones are Gunter's Space Page [17] and Earth Observation Portal [18] or from the same Spacetrak ${ }^{\mathrm{TM}}$ database (e.g.: previous satellites of the same bus type, same constellation, etc.). In addition, the geometry of the spacecraft has been added since this is needed for the calculation of the drag surface (Table 1).

\subsection{STELA tool}

STELA reflects the standard concerning the protection of LEO and GEO regions (lifetime and protected regions crossing of disposal orbits). The software allows efficient long-term propagation of LEO, GEO, and GTO types orbits based on semi-analytical models, statistical analysis and assessment of protected regions criteria.

The main input parameters (general) for the performed STELA simulations to understand the future scenario are the following:

- Orbit parameters (zp, za, $i, \Omega, \omega, M$ ): mean values in the reference frame celestial mean of date (MOD).

- Initial date: the future launch date has been set.

- Object characteristics: total mass of the spacecraft, mean cross-sectional area used to compute the solar radiation pressure force (reflectivity area) and the atmospheric drag force (drag area), the reflectivity coef-
Table 2 Advanced parameters settings for STELA simulations performed

\begin{tabular}{ll}
\hline Algorithms & Settings \\
\hline Integrator step & $24 \mathrm{~h}$ \\
Atmospheric drag quadrature points & Enabled \\
Solar radiation pressure (SRP) & Enabled \\
Third body perturbations (Sun and Moon) & Enabled \\
Earth perturbation & Zonal order $7 \times 0$ \\
Re-entry altitude & Default $120 \mathrm{~km}$ \\
\hline
\end{tabular}

ficient (1.5 mean value suggested in [19] for LEO), and the drag coefficient (constant value of 2.2).

- Atmospheric model: empirical model NRL-MSISE-00 (already set in STELA) for the density calculation.

- Solar activity: variable solar flux vs time, this includes the future mean prediction given by NOAA and NASA till 2318 [20].

Regarding "parameters advanced section" the default parameters, already set in STELA have been adopted (Table 2).

The computation of mean cross-sectional area has been obtained with STELA Mean Area Tool drawing a simplified model of the spacecraft considering the Random tumbling orientation model.

Before analysing the future scenario, we evaluated the confidence of STELA for de-orbit simulations, particularly in situations of uncertainty in the spacecraft geometry. The purposes of this analysis were the comparison of real data (TLE) from already decayed and re-entered satellites (without any propulsion, just natural decay), with respect to STELA simulation propagation; the understanding of the influences of orbit parameters and perturbations; the proper configuration for geometry to be adopted (Table 3 ).

The TLEs for the decayed satellites have been provided by the NORAD Two-Line Element Sets Current Data via Data Request [21]. The case studies are all Micro and Minisatellites.

As can be seen from the Figs. 2 and 3, the simulation trend is conservative (more time needed for re-enter) with respect to the real data, this gives confidence and margins for the results on future simulation.

The error on time for Uosat- 1 and OPS7353, both in sun-synchronous orbits, is near or even below the $5 \%$. The error encountered in the simulation is related to different uncertainties; one of them is the atmospheric model accuracy prediction of the density. The NRL-MSISE-00 is influenced by the accuracy of the future solar cycles and geomagnetic activity used as inputs. The density uncertainty is of $15 \%$ for mean activity conditions, as stated in [5]. 
Table 3 Mission case studies with spacecraft mass and main orbit parameters

\begin{tabular}{llllll}
\hline Mission & Orbit type & Mass $(\mathrm{kg})$ & $\begin{array}{l}\text { Perigee } \\
(\mathrm{km})\end{array}$ & $\begin{array}{l}\text { Apogee } \\
(\mathrm{km})\end{array}$ & $\begin{array}{l}\text { Inclination } \\
\left({ }^{\circ}\right)\end{array}$ \\
\hline Uosat-1 & SSO & 52 & 532 & 537 & 97.46 \\
Tiros-02 & LEO & 127 & 609 & 744 & 48.56 \\
OV1-10 & SSO & 130 & 631 & 758 & 93.41 \\
OPS7353 & SSO & 130 & 527 & 751 & 99.01 \\
\hline
\end{tabular}

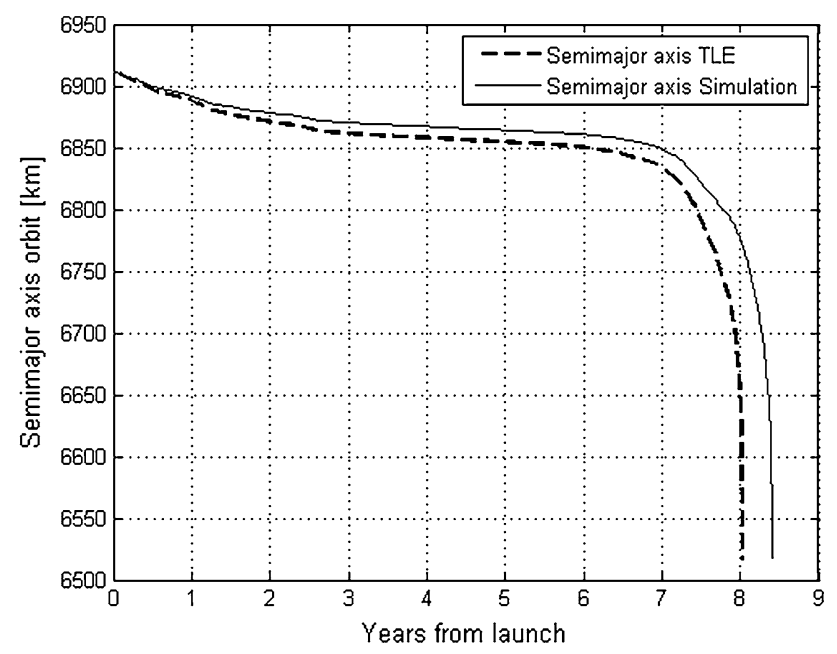

Fig. 2 Decrease of semi-major axis of Uosat-1 during years, real satellite data (dashed) and STELA simulation (continuous)

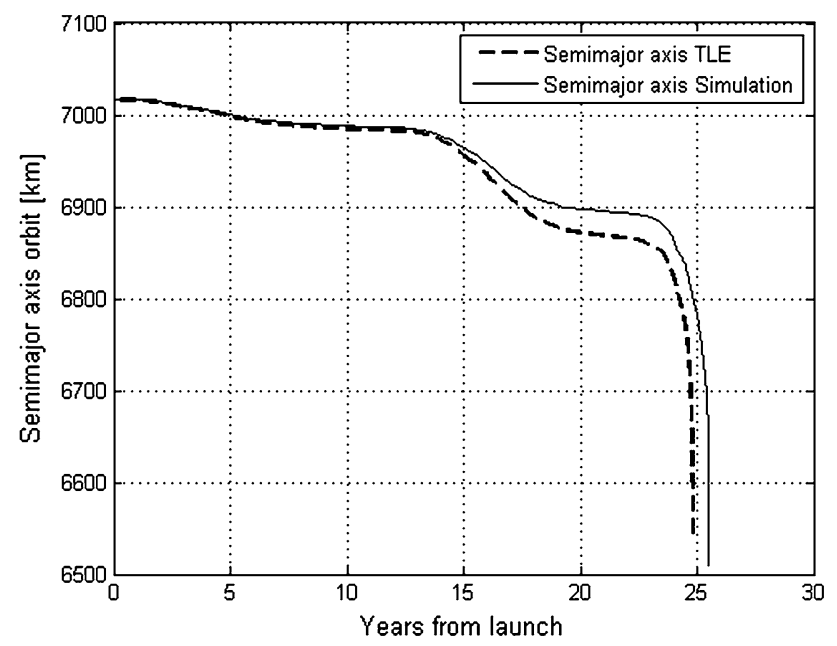

Fig. 3 Decrease of semi-major axis of OPS7353 during years, real satellite data (dashed) and STELA simulation (continuous)

Another uncertainty is the cross-sectional area for atmospheric drag that can vary over time depending on the orientation of the $\mathrm{S} / \mathrm{C}$, while for the simulations, random tumbling mean values have been adopted. The error due to the area uncertainty is very evident for OV1-10, in this case there was high uncertainty in the geometry data (lack of information for this military satellite) and consequently the value computed for the drag area was not very reliable.

In the case of Tiros-02, which had an orbit inclination of $48.5^{\circ}$, the simulation underestimates the real TLE data, despite the simulation time is just $10 \%$ shorter than the real time. However, this satellite fits in the critical inclination group, identified in STELA in the ranges $40^{\circ}-80^{\circ}$ and $110^{\circ}-130^{\circ}$ with most sensitive inclinations close to $41.6^{\circ}$ [22] (Table 4).

These inclinations lead to resonance effects due to various perturbations with significant effect on the lifetime. STELA recommendation for this inclination range is running several simulations and process them statistically [23]. This is relevant to consider for the de-orbit propagation of future satellites if they fall in this group.

\section{Future scenarios}

In this section, the results obtained from the performed STELA simulations for the de-orbit analysis of future satellites are presented. The satellites considered are planned to be launched in the time frame 2015-2020 (launch date stated) and they are divided into different orbit subgroups (SSO, polar, LEO general) as mentioned before.

The main aim of this work is helping in defining toplevel requirements for the design of passive devices to deorbit a range of different spacecraft at the end of life; for this reason, the satellites have been divided into classes depending on their mass. Medium (>1000 kg) and larger classes have not been considered since they are out of the purpose of this study. These S/C are out of the target for passive drag devices, because they are already equipped with propulsion subsystem and they need a controlled reentry, as some components (e.g., titanium tanks) are most likely to survive the re-entry.

\subsection{Current situation}

The current situation, presented in Fig. 4, identifies the de-orbit time compliance taking into account only the future launches for LEO and not the satellites already on-orbit.

This study is performed by considering the natural decay of these future missions, as its purpose is to identify which missions would need to employ de-orbit technologies. It is noted that some of the future missions may be already intending to either use a de-orbit device or the satellite's propulsion to achieve de-orbit.

The column chart presented shows the results for the mass classes: pico, nano, micro, mini and small. 
Table 4 Relative error on time (years) for the simulation with reference to the real value at different semi-major axis during decay

\begin{tabular}{lllll}
\hline Semimajor axis $(\mathrm{km})$ & Uosat-1 & Tiros-02 & OV1-10 & OPS7353 \\
\hline Initial-5 km & $17.54 \%(6907 \mathrm{~km})$ & $-1.00 \%(7050 \mathrm{~km})$ & $37.51 \%(7068 \mathrm{~km})$ & $11.11 \%(7012 \mathrm{~km})$ \\
6878 & $43.17 \%$ & $-3.22 \%$ & $65.63 \%$ & $24.82 \%$ \\
6828 & $4.36 \%$ & $-15.09 \%$ & $66.13 \%$ & $2.72 \%$ \\
6778 & $5.19 \%$ & $-12.69 \%$ & $65.93 \%$ & $2.46 \%$ \\
6728 & $4.35 \%$ & $-10.40 \%$ & $65.96 \%$ & $2.60 \%$ \\
6675 & $4.47 \%$ & $-9.55 \%$ & $66.01 \%$ & $2.83 \%$ \\
6628 & $4.66 \%$ & $-9.37 \%$ & $66.07 \%$ & $2.78 \%$ \\
Final data & $4.75 \%(6516 \mathrm{~km})$ & $-9.33 \%(6562 \mathrm{~km})$ & $66.11 \%(6516 \mathrm{~km})$ & $2.80 \%(6539 \mathrm{~km})$ \\
\hline
\end{tabular}

Fig. 4 Compliance with 25 years re-entry for planned satellites 2015-2020 in LEO for all the orbit type categories: SSO, polar, LEO general. Assumes no specific de-orbit measures are used. Note that OneWeb constellation of 700 LEO satellites has not been included in the study (OneWeb states that these satellites will be designed to be compliant with debris guidelines)

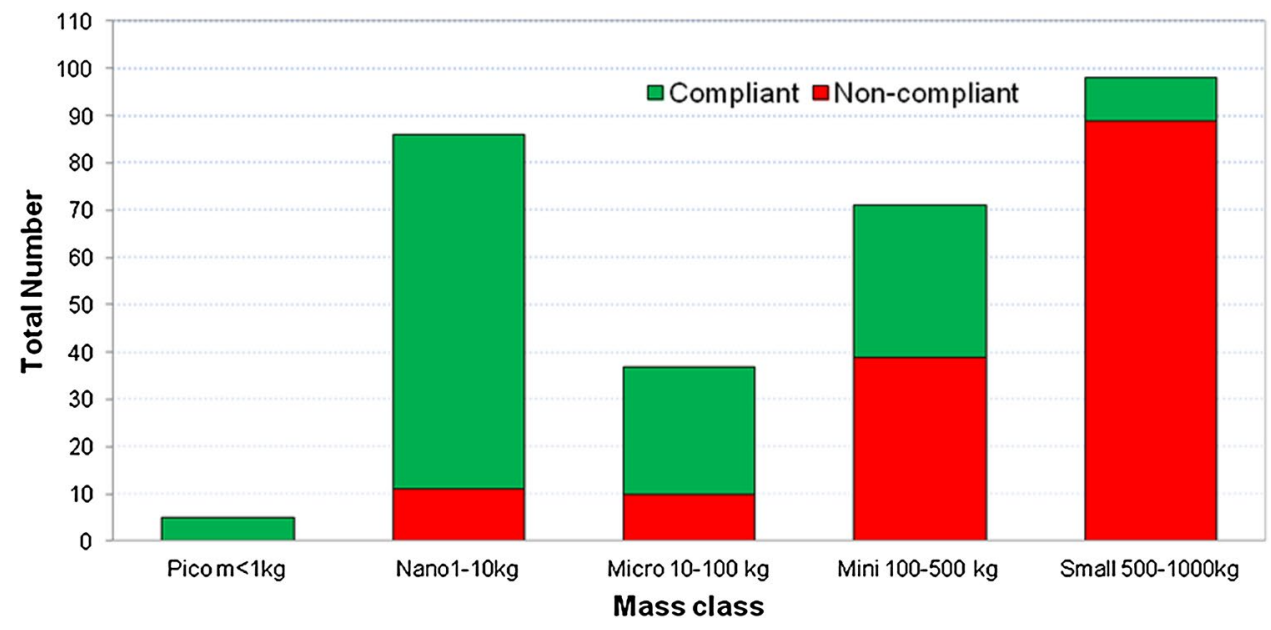

As can be seen in the figure, picosatellites are not relevant as the amount is not significant and anyway they comply. The nanosatellites, in this group fit the cubesats, are generally compliant as usually they are in very low earth orbit (e.g.: launched from the ISS); however, $15 \%$ of them will require some kind of passive device to re-enter within the 25 years. Furthermore, more attention shall be paid on this class considering the potential increase in the coming years confirmed by different projections, SpaceWorks foresees a compound annual growth rate (CAGR) equal to $23.8 \%$ average [24].

The microsatellites non-compliant are comparable in number to the nanosats but the mass is significantly heavier and so the potential impact they could have as source of debris is greater; another aspect to take into account is that most of them don't have any propulsion subsystem.

More than $50 \%$ of the minisatellites will not comply with the requirements, which makes them an interesting target for the passive devices, also considering that their propulsion capabilities are limited. In addition, the drag device is an attractive option considering the relative low additional mass respect to the fuel mass needed to re-enter.

The small satellites are the less compliant to the re-entry time; however, most of them (probably all) are equipped with propulsion subsystem and the higher quantity is related to the presence of satellite constellations (e.g.,
Iridium-NEXT on polar orbit). Nevertheless, some passive solutions can be implemented as back-option.

The bubble charts (Figs. 5, 6, 7) show a more detailed insight of the compliance distribution depending on the altitude and mass in the different orbit subgroups. It can be seen clearly where there will be a concentration of satellites in the next years.

Looking at the SSO chart, a sparse distribution with different variety of spacecraft is identified. A constellation of 14 satellites is evident at $500 \mathrm{~km}$; this is the Skybox constellation which results compliant if the target altitude is confirmed. Overall, on sun-synchronous orbits, half of the satellites will be compliant with the 25 years and half of them not; so de-orbit methods with scalable design are needed.

For polar orbits, the situation is different since fewer satellites fall in this group; however, this orbit type is mostly used for communication satellites. The main constellation is in fact Iridium-NEXT (72 satellites to substitute the old Iridium), non-compliant if no de-orbit methods are implemented. In addition, the presence of four other satellites can be observed at the same altitude, this is the Ionosfera spacecraft constellation.

The last plot shows the LEO satellites which don't fit with the previous subgroups. For this group, the results presented consider only $\mathrm{S} / \mathrm{C}$ with mass below $300 \mathrm{~kg}$. It has 
Fig. 5 Distribution of satellites' compliance with 25 years re-entry considering mass and altitude for sun-synchronous orbits, the size of the bubble represents the satellites number (number is 1 if not shown). Assumes no specific de-orbit measures are used

Fig. 6 Distribution of satellites' compliance with 25 years reentry considering mass and altitude for polar orbits. Assumes no specific de-orbit measures are used

Fig. 7 Distribution of satellites' compliance with 25 years reentry considering mass below $300 \mathrm{~kg}$ and altitude for LEO (non-SSO or polar orbit type). Assumes no specific de-orbit measures are used
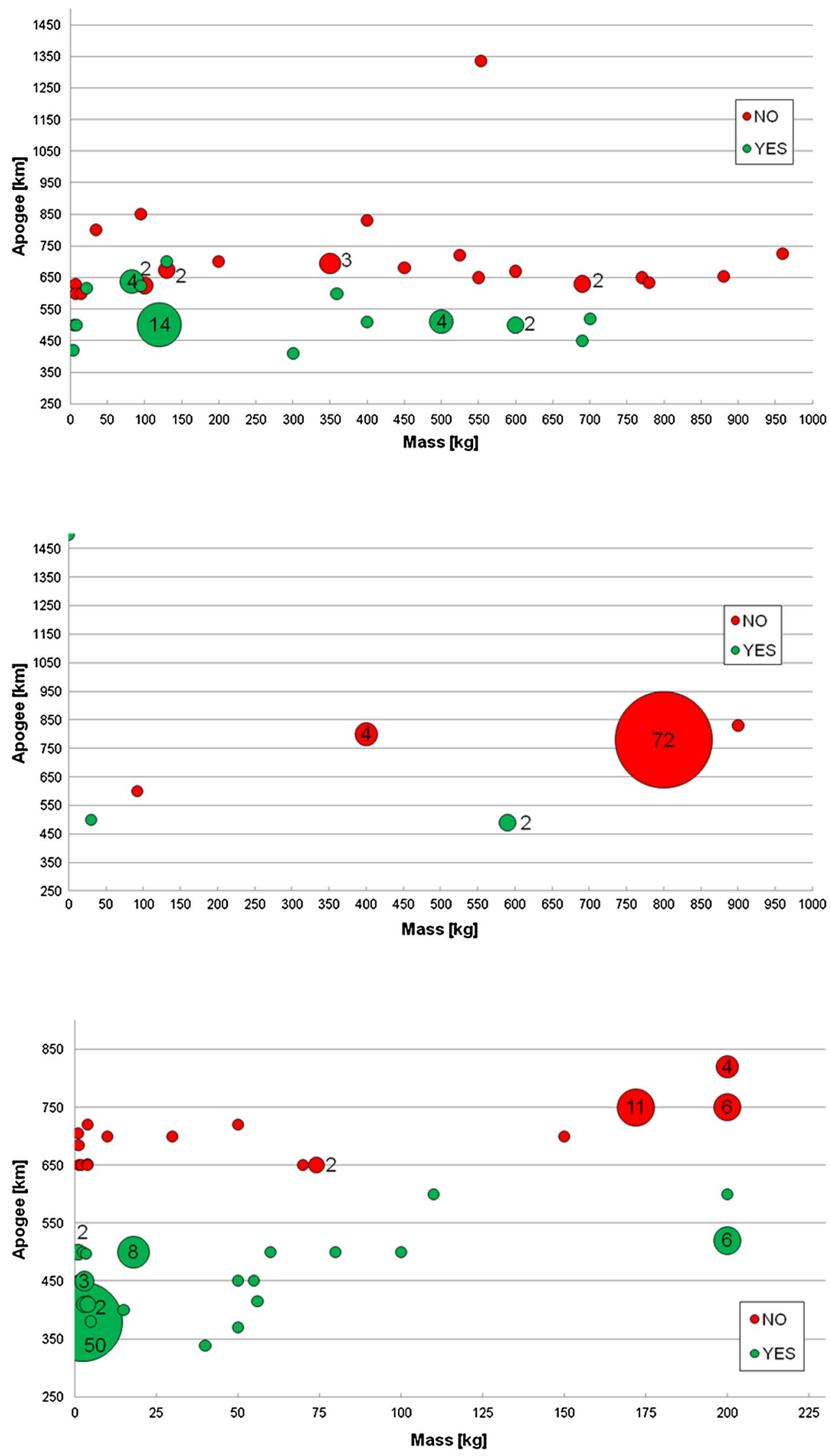
been decided to focus on lighter masses for two reasons: firstly there are only few satellites with heavier masses, 4 of them around $400 \mathrm{~kg}$ are compliant and 6 Globalstar-SG are not but the drag effect is not significant on the planned altitude (around $1400 \mathrm{~km}$ ); secondly, in this way, a more accurate scenario is depicted.

There's a noticeable concentration of nanosatellites, mostly cubesat type (QB50 with $50 \mathrm{~S} / \mathrm{C}$ and EDSN-cubesats with $10 \mathrm{~S} / \mathrm{C}$ ), around $400 \mathrm{~km}$ and easily compliant due to the very low altitude. Another constellation in line with the re-entry requirement is CYGNSS with 8 microsatellites at $500 \mathrm{~km}$ of altitude.

More of interest for the purpose of this work is looking at the concentration of non-compliances. Three main groups can be identified: ORBCOMM OG2 with 11 new satellites, FORMOSAT constellation with 6 satellites at $750 \mathrm{~km}$ (and the other half compliant at $500 \mathrm{~km}$ ), and the 4 Russian MKA-FKI satellites at $800 \mathrm{~km}$. In addition, it is relevant to note how many different nanosats and microsats will be positioned in higher orbit than the commonly used one (see green bubbles for comparison) and so they will need drag disposal strategies.

\subsection{Design requirements}

The outcomes of the future launch scenario show that the population of satellites that needs de-orbit methods spreads across different mass classes and buses. The key drivers for the design of passive drag device are then to be scalable (to be suitable for wide range of satellite size), flexible (to adapt to different satellite configuration), and easy to manufacture, assemble, and test.

In the preliminary phase of the design, the size of the sail needed to meet the 25 years requirement and the mechanical configuration of the device (type of mechanism) must be clear. The sail area depends on the S/C mass and crosssectional area, and on the mission orbit (not only altitude but also inclination). Considering the orbit altitude, the same sail size can be used as standard to de-orbit similar S/C but at different altitudes, in Fig. 8 can be seen the years needed to reenter with different sail configurations varying the altitude.

The mechanical configuration (envelope, mass, interfaces) of the mechanism itself can be modular and have available two or three different options depending on the satellite class; for example, having a self-contained unit for nanosats and light microsats such as the DOM, and, instead, an external frame on the panel side of the spacecraft such as Icarus-1 on TechDemoSat-1 for heavier satellites.

Other relevant properties needed for the design of the device are the ADCS architecture and so the type of stabilisation for the S/C, power bus architecture and the location availability for positioning the de-orbit device.

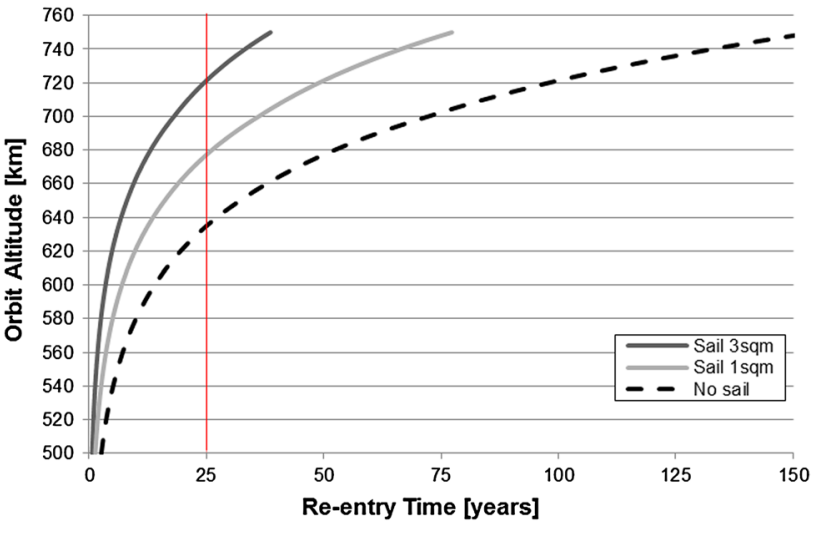

Fig. 8 Lifetime for $100 \mathrm{~kg} \mathrm{~S} / \mathrm{C}$ with cross-sectional area of $1 \mathrm{~m}^{2}$ (without sail) with different drag area. Lifetime calculations with solar average values and linear variation of density and scale height

\section{Case study: DOM on ESEO}

\subsection{ESEO}

ESEO is a microsatellite mission to low earth orbit, according to ALMASpace ESEO mission analysis report 2013, the target orbit is a circular sun-synchronous orbit with 10:30 LTAN and an altitude of $523 \mathrm{~km}$. ESEO is an ESA Education Office project with SITAEL (former ALMASpace) as industrial prime contractor and 7 payloads expected on-board being developed, integrated, and tested by teams of European university students.

The primary objective is indeed to give European students hands-on space project experience [25]. The ESEO satellite has the following mission objectives:

1. to take pictures of the Earth and/or other celestial bodies from Earth orbit for educational outreach purposes;

2. to provide dosimetry and space plasma measurement in Earth orbit and its effects on satellite components;

3. to test technologies for future satellite missions.

The de-orbit mechanism (DOM) fits in the realisation of the third objective.

\subsection{De-orbit mechanism}

A case study of a passive strategy is given by the De-Orbit Mechanism (DOM) technological demonstrator which is currently under development at Cranfield University.

The device is designed to deploy a drag sail at the end of the satellite mission thus enlarging the effective satellite area and hence, allowing the satellite an accelerate orbit decay and re-entry back to Earth. The design is based on Cranfield Space Research Centre experience in de-orbit sails (e.g., Icarus 1 on the TechDemoSat-1, launched in 2014). 


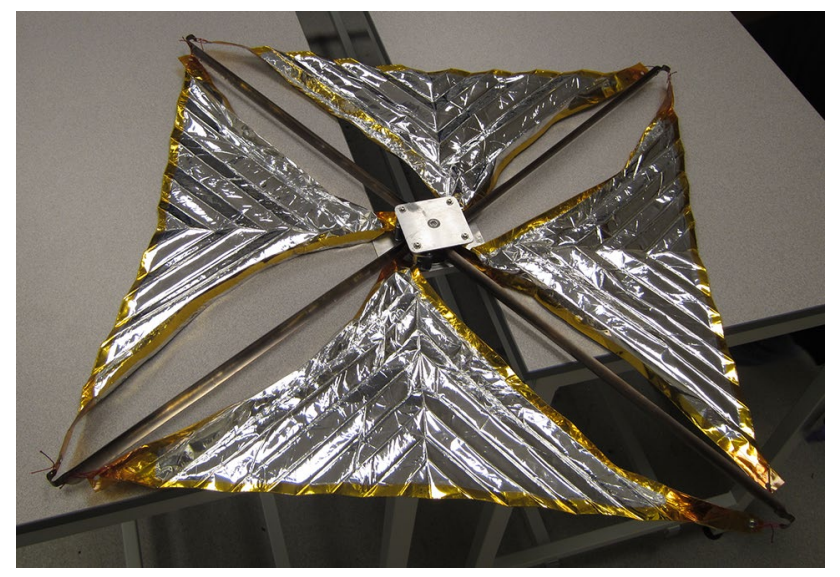

Fig. 9 DOM EBB deployed

The DOM is a self-contained unit (volume envelope $140 \mathrm{~mm} \times 80 \mathrm{~mm} \times 56 \mathrm{~mm}$ ) of less than $0.5 \mathrm{~kg}$, mounted on the side panel of the ESEO satellite. It houses tape spring booms, aluminized Kapton sails (each of $0.125 \mathrm{~m}^{2}$ ), and a release mechanism (Figs. 9, 10).

The booms, which are made of copper-beryllium, and the sails, with a pattern developed from bio-mimetic "Miura-ori" studies [26], are rolled up around a central spool in the middle of the device and held in position by Kevlar cords. Thus, the device is compactly stored in a single unit before actuation. Once the deployment-command is sent, by closing a series of relay switches and thereby activating two CYPRESTM cord cutters, the Kevlar cords are cut and the strain energy, stored in the boom arms during the coiling process, is transferred into kinetic energy about the central spool, resulting in deployment.

The CYPRESTM cord cutters are propellant generated pyrotechnical parts with qualification heritage from Icarus-1 de-orbit sail [12]; they are also independently qualified for use in human life-critical reserve parachute release devices.

The copper-beryllium booms deploy with a constant torque of the order of $0.1 \mathrm{Nm}$, this is the steady-state bending moment calculated for a tape spring coiled onto a spool of radius approximately equal to its curvature [27]. In this way the booms fold naturally and jamming is prevented during deployment.

Deployment tests were performed in two cases: (1) at $-33{ }^{\circ} \mathrm{C}$ after the unit has been tested at $-80{ }^{\circ} \mathrm{C}$ in a climatic chamber; (2) after vacuum test, which simulated the depressurization expected during the launch phase. In the second case the deployment was performed after the vacuum test due to size constraints of the vacuum chamber; the main purpose of the test was to ensure that the depressurisation has no billowing effect on the sails. Both the tests showed nominal deployment of the sails.

Considering the DOM is a technological demonstrator, and highly over engineered, the resistive torque due to the sail

Fig. 10 DOM exploded view

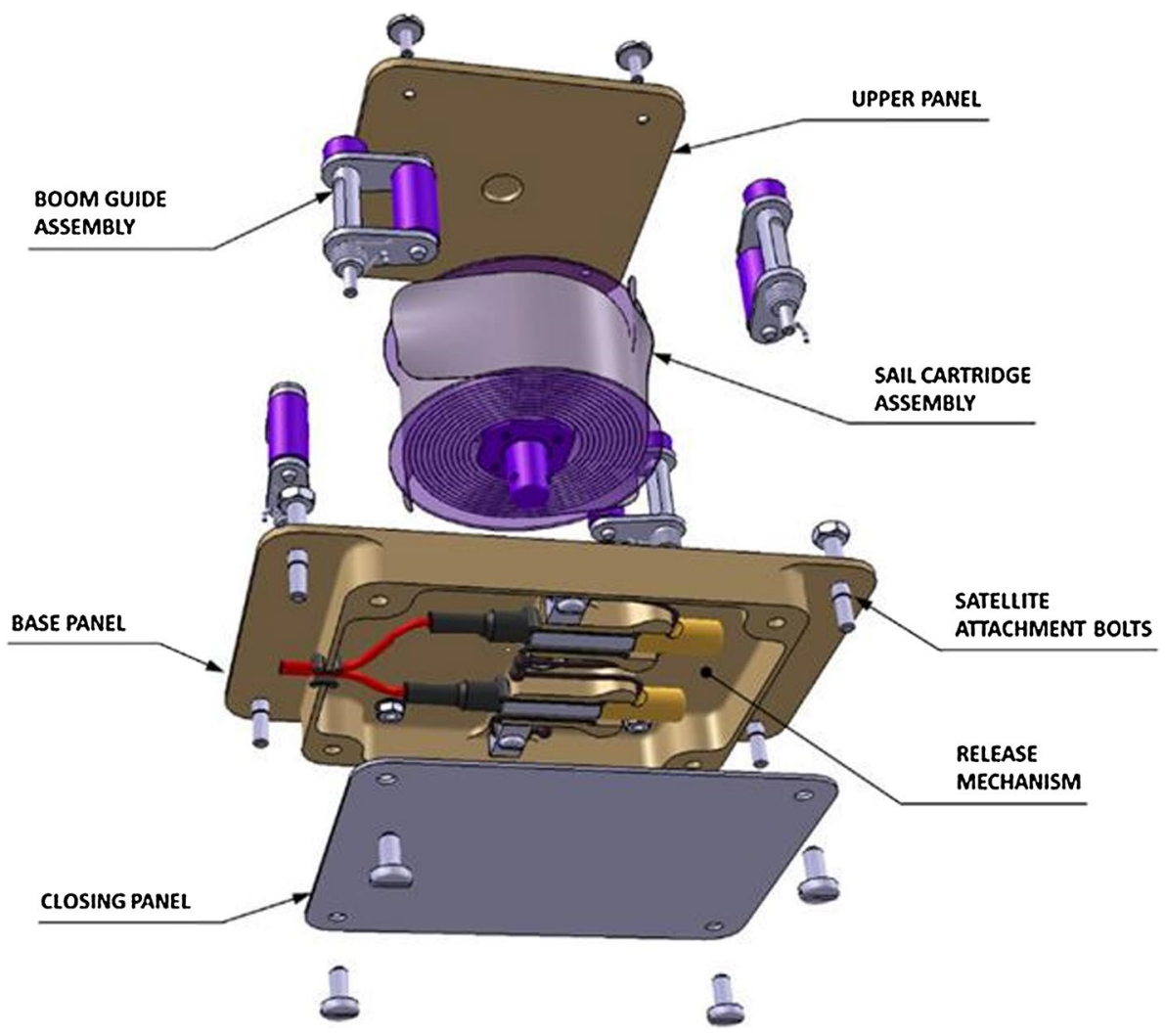


unrolling has not been measured; this will be addressed in further experimental test evaluating the scalability of the design.

The goal is to reach a final design capable of de-orbiting a range of satellites with no need of significant re-engineering. The DOM is currently on phase D of the project, with the CDR milestone successfully achieved in 2015.

\section{Conclusions}

The future market for passive de-orbit devices in LEO has been investigated in this paper. The need of passive de-orbit strategies shows a potential considering the recent adoption of ISO 24113 by ESA and taking into account the growing of small classes of satellites in the next years.

The process of filling the database gaps has not been an easy task as this has relied on the information publicly available, so despite a good amount of data the numbers are underestimated. STELA has been confirmed a valid tool for de-orbit analysis, in particular considering the uncertainty in the S/C geometry and on-orbit perturbation given by the models. Two mass categories stand out as target for the passive devices: micro and minisatellites, with back-up option to be considered for the small satellites. A design based on the DOM can be applied on light microsats and nanosatellites.

Acknowledgments The de-orbit mechanism work is part-funded by the European Space Agency Education Office under the ESEO project, managed by the Industrial System Prime Contractor SITAEL (former ALMASpace). In coordination with the ESA Education Office, they provide system level and technical support to the Cranfield team. The support is gratefully acknowledged.

\section{Compliance with ethical standards}

Conflict of interest The authors declare that they have no conflict of interest.

Open Access This article is distributed under the terms of the Creative Commons Attribution 4.0 International License (http://creativecommons.org/licenses/by/4.0/), which permits unrestricted use, distribution, and reproduction in any medium, provided you give appropriate credit to the original author(s) and the source, provide a link to the Creative Commons license, and indicate if changes were made.

\section{References}

1. ESA: Clean space. http://www.esa.int/Our_Activities/Space_ Engineering/Clean_Space. Accessed 15 Jan 2014

2. ESA Director General's Office: ESA/ADMIN/IPOL(2014)2_ Space Debris Mitigation for Agency Projects (2014). http:// www.iadc-online.org/index.cgi?item=documents. http://www. iadc-online.org/References/Docu/admin-ipol-2014-002e.pdf. Accessed 13 Apr 2015

3. Committee on the Peaceful Uses of Outer Space: Updated set of draft guidelines for the long-term sustainability of outer space activities. Report No.: A/AC.105/C.1/L.340 (2014)
4. BSI Standards Publication: ISO 24113:2011 Space systemsspace debris mitigation requirements (2011). https://extranet. cranfield.ac.uk/Bibliographic/BibliographicInfoData/,DanaInf o=bsol.bsigroup.com,SSL +000000000030233881. Licenced Copy Cranfield University, 15 April 2015

5. ESA Space Debris Mitigation WG: ESSB-HB-U-002, Distribution: ESA, pp. 1-95 (2015)

6. IADC: IADC space debris mitigation guidelines. In: Steering Group and Working Group 4 (2007)

7. Rossettini, L.: D-Orbit-Solution for Our Future. Technical Day on De-Orbiting Strategies. ESTEC (2015). https://indico.esa.int/ indico/event $/ 73 /$ material/0/. Accessed 3 June 2015

8. Taylor, R., Hobbs, S.: Conceptual development of a de-orbiting payload for micro-satellites in LEO. http://cclibweb-3.central. cranfield.ac.uk/handle/1826.1/7952. Accessed Apr 2015

9. Castet, J.F., Saleh, J.H.: Satellite and satellite subsystems reliability: statistical data analysis and modeling. Reliab. Eng. Syst. Saf. 94, 1718-1728 (2009)

10. Castet, J.-F., Saleh, J.H.: Beyond reliability, multi-state failure analysis of satellite subsystems: a statistical approach. Reliab. Eng. Syst. Saf. 95, 311-322 (2010)

11. Nock, K.T., Aaron, K.M., McKnight, D.: Removing orbital debris with less risk. J. Spacecr. Rockets 50, 365-379 (2013)

12. Kingston, J., Hobbs, S., Roberts, P., Juanes-Vallejo, C., Robinson, F., Sewell, R., Snapir, B., Llop, J.V., Patel, M.: Use of CYPRESTM $^{\text {TM }}$ cutters with a Kevlar clamp band for hold-down and release of the Icarus De-Orbit Sail Payload on TechDemoSat-1. Acta Astronaut. 100, 82-93 (2014)

13. Harkness, P., McRobb, M., Lützkendorf, P., Milligan, R., Feeney, A., Clark, C.: Development status of AEOLDOS-a deorbit module for small satellites. Adv. Sp. Res. 54, 82-91 (2014)

14. Visagie, L., Lappas, V., Erb, S.: Drag sails for space debris mitigation. Acta Astronaut. 109, 65-75 (2015)

15. Seradata SpaceTrak: SPACETRAK user guide v1.4. http://www. seradata.com/. Accessed Jan 2015

16. Fraysse, H., Morand, V., Le Fevre, C., Deleflie, F., Wailliez, S., Lamy, A., Martin, T., Perot, E.: Long term orbit propagation techniques developed in the frame of the french space act. J. Aerosp. Eng. Sci. Appl. 4, 1-16 (2012)

17. Gunter's Space Page. http://space.skyrocket.de/. Accessed 5 Apr 2015

18. ESA: Earth Observation Portal. https://eoportal.org/web/eoportal/satellite-missions. Accessed 1 Jan 2015

19. Wertz, J.R., Everett, D.F., Puschell, J.J.: Space Mission Engineering: The New SMAD. Microcosm Press, Hawthorne (2011)

20. CNES: STELA user's guide, Version 2.5 (2013)

21. Kelso, T.S.: NORAD two-line element sets current data. http:// www.celestrak.com/NORAD/elements/. Accessed 1 Dec 2014

22. Lamy, A., Morand, V., Le Fevre, C., Fraysee, H.: Resonance effects on lifetime of low earth orbit satellites. In: 23rd International Symposium on Space Flight Dynamics, Pasadena (2012)

23. Le Fèvre, C., Fraysse, H., Morand, V., Lamy, A., Cazaux, C., Mercier, P., Dental, C., Deleflie, F., Handschuh, D.A.: Compliance of disposal orbits with the French space operations act: the good practices and the STELA tool. Acta Astronaut. 94, 234-245 (2014)

24. Buchen, E., DePasquale, D.: 2014 Nano/Microsatellite Market Assessment. SpaceWorks Enterprises, Inc. (SEI), Atlanta (2014)

25. ESA Education: ESEO mission. http://www.esa.int/Education/ ESEO_mission. Accessed 1 Dec 2014

26. Tanizawa, K., Miura, K.: Large displacement configurations of bi- axially compressed infinite plate. Trans. Jpn. Soc. Aeronaut. Space Sci. 20(5), 177-187 (1978)

27. Seffen, K.A., Pellegrino, S.: Deployment dynamics of tape springs. Proc. R. Soc. A Math. Phys. Eng. Sci. 455, 1003-1048 (1999) 